\title{
Editorial
}

\section{Latex allergy}

Michael E. Weiss MD, ${ }^{*}$ Carol A. Hirshman MD $\dagger$
Natural rubber (latex) is a milky juice obtained from the sap tree, Hevea brasiliensis, that grows in the Amazon region. During the manufacturing process, various small molecular weight chemicals may be added before the latex is vulcanized (cured with heat and sulphur $-130^{\circ} \mathrm{C}$ for 5-30 min). These small molecular weight chemicals (accelerators, curing agents, antioxidants, stabilizers, dyes, bleaches, retarders, and blowing products) help to give natural rubber its desired final properties, such as colour, texture and elasticity. Proteins make up 2-3\% of the final latex product. These proteins are water soluble and heat and chemically stable. Latex is presently used in over 40,000 products and is a component of many medical devices, including gloves, urinary catheters, condom catheters, intubation tubes, anaesthesia masks, dental cofferdams, rebreathing masks, barium enema cuffs (recently recalled), ostomy bags, and medication stoppers.

It has long been known that latex exposure could cause contact dermatitis. Contact dermatitis is caused by a type $4, T$ cell mediated, delayed hypersensitivity allergic reaction. Small molecular weight chemicals (accelerators or antioxidants) added during the manufacturing process act as haptens and trigger-sensitized $\mathrm{T}$ lymphocytes to recruit and stimulate the proliferation of other lymphocytes and mononuclear cells. This ultimately leads to tissue inflammation and dermatitis. ${ }^{\prime}$

It was not until 1979 that the first case of contact urticaria due to latex was reported. ${ }^{2}$ Since then, latex has been shown to cause not only contact urticaria, but also generalized urticaria, asthma, and anaphylaxis, which was first reported in $1987 .{ }^{3}$ More than ten deaths have been attributed to latex anaphylaxis. ${ }^{4}$ The cause of these reactions is an IgE antibody directed against one or more proteins in the latex. ${ }^{5}$ In patients with this anti-latex IgE antibody, latex exposure may lead to a type 1 , immediate hypersensitivity allergic reaction resulting in mast cell and basophil degranulation and potentially life-threatening anaphylaxis.

Certain population groups are at increased risk for

From the *Department of Medicine, University of Washington, Division of Allergy, Seattle, Washington and the $†$ Department of Anesthesiology, The Johns Hopkins Medical Institutions, Baltimore, Maryland. developing anaphylactic reactions to latex. These include health care workers, especially those with frequent exposure to latex gloves. Studies have shown that $6-10 \%$ of operating room medical personnel have IgE antibodies directed against latex proteins. ${ }^{6,7}$ Another group at very high risk are children with myelomeningocele or congenital urologic abnormalities. ${ }^{8-10}$ In fact, this group of patients may be at greatest risk for severe latex reactions. In one study involving five myelodysplasia clinics, 187 families were questioned and $25 \%$ of the children with myelomeningocele had a positive history of allergic reactions to latex products. " Investigators have found that $71 / 127(56 \%)$ patients with myelomeningocele have IgE antibodies to latex. ${ }^{12-14}$ It is thought that repetitive exposure to latex products (gloves, catheters, etc.) at an early age from frequent urinary catheterization and multiple surgical operations and procedures leads to latex sensitization.

Patients with atopy (allergic rhinitis, asthma, or atopic dermatitis) are also more likely to develop latex reactions. ${ }^{8,9,15}$ In addition, individuals with hand eczema who are exposed to latex gloves are at high risk for latex anaphylaxis. ${ }^{15,16}$ Whether eczema, by disrupting the natural skin barrier, allows latex antigens to better reach immunocompetent cells below the epidermis and therefore increase the chance for latex sensitization, or whether eczema, itself, is an early manifestation of latex allergy, is presently unknown. Patients with banana allergy appear to be at increased risk for latex reactions. ${ }^{17}$

What, then, can be done to minimize the risk of latex reactions during anaesthesia? First, awareness of latex allergy and the population groups at increased risk for such reactions needs to be disseminated. During the pre-anaesthetic evaluation, specific questions concerning previous reactions to latex or rubber products should be asked. ${ }^{18}$ Patients who have suffered systemic, severe intraoperative reactions to latex often have a history of contact urticaria or angioedema when exposed to rubber products such as gloves, rubber balloons, latex condoms, diaphragms, or pacifiers. Latex allergy should be suspected in patients who have had previous unexplained intraoperative anaphylaxis. In a recent study, $5 / 43(10 \%)$ cases of intraoperative anaphylaxis at one hospital were found to be caused by latex allergy. ${ }^{19}$ The temporal pattern of intraoperative anaphylaxis due to latex is often different from 
anaphylaxis secondary to anaesthetic agents. Intraoperative anaphylaxis due to latex allergy often occurs $20-150 \mathrm{~min}$ after induction, probably when latex-gloved hands contact mucosal and peritoneal surfaces, leading to systemic absorption of latex proteins. ${ }^{20}$ This is in contrast to reactions related to anaesthetic agents, which typically occur at the time of induction.

Confirmation of latex allergy should be obtained by doing latex epicutaneous skin tests using either natural latex obtained from latex manufacturing companies, an extract prepared by incubating latex gloves in phosphatebuffered saline, or by skin testing through a moistened latex glove. ${ }^{16,21,22} \mathrm{~A}$ commercially available skin test reagent (natural latex) has recently become available in Canada (Bencard Lab., Mississauga, Ontario). Unless one is quite familiar with all aspects of allergy skin testing, the patient should be referred to an allergist for such tests and their interpretation. Epicutaneous skin testing using serial dilutions of latex has a high safety profile and allows one to observe a dose-dependent sensitivity.

Intradermal tests are generally not needed and have provoked systemic reactions in some patients. A provocative challenge (use test) where the individual wears a moistened latex glove or glove finger has also resulted in systemic reactions and generally is not recommended. A commercial latex RAST (Latex disc 82, Pharmacia Diagnostics, Piscataway, $\mathrm{NJ}$ ) is available but is not as sensitive as epicutaneous skin testing. The latex RAST may be negative between $20-45 \%$ of the time in patients with positive skin tests to latex. ${ }^{15,23}$ Recently, there have been a few case reports of patients who have had systemic reactions to epicutaneous skin tests. Therefore, it may be prudent to first do a latex RAST and if negative proceed to epicutaneous skin tests.

If patients require surgery and have confirmed latex sensitivity, or have convincing histories of latex reactions, then avoidance of latex products, especially latex gloves, is mandatory. Strong consideration should also be given to latex avoidance in all patients with myelodysplasia or congenital urological abnormalities, since the prevalence of latex sensitization appears to be so high in this population.

Avoidance of latex gloves can be achieved using synthetic rubber gloves made from material such as Neolon (neoprene) (Deseret Medical, Inc.) or Elastyren (styrene butadiene) (Hermal Pharmaceutical Labs, Inc.). Since synthetic gloves are expensive, may have properties which make their use less desirable, and are not always readily available, medical personnel caring for latex allergic patients can also use vinyl gloves taped in place at the wrist over washed latex gloves. One study suggested that thorough washing of latex gloves prior to their use in latex allergic patients was sufficient to remove the allergenic antigen, ${ }^{22}$ but until confirmatory studies are done, this is not recommended.

Preoperative treatment of latex allergic patients with steroids and antihistamines has been suggested without any evidence of efficacy. Since pretreatment has never been demonstrated to prevent IgE mediated allergic reactions and since pretreatment may mask early signs of anaphylaxis, its use is not generally recommended. The anaesthetist must monitor for early signs of anaphylaxis and must be ready to aggressively treat a reaction, should it occur.

Since we have become a very mobile society, the patient with latex allergy must be instructed to mention this condition to all future health care providers. Latex allergic patients should wear medic-alert information, should be given an Epi Pen and instructed in its use, and should carry various sizes of non-latex gloves for potential emergency use.

While latex and rubber products have been used for many years, it is only in the last decade that reports of contact urticaria and systemic anaphylactic reactions to latex have been described. It is possible that, in the past, the connection of latex exposure to these reactions was not made, but this is unlikely. It is also possible that the recent increased need for latex products, especially gloves and condoms, because of the HIV epidemic, has resulted in increased exposure and sensitization. It is also possible that a change in the manufacturing process has led to increased antigenicity of latex products.

\section{Allergie au latex}

Le caoutchouc naturel ou latex est une sèvre laiteuse provenant de l'Hévéa brasiliensis, un arbre de la forêt amazonienne. Au cours de la transformation manufacturière du latex, des produits chimiques de petit poids moléculaire sont ajoutés avant la vulcanisation de la matière première (traitement au sulfure et à la chaleur à $-130^{\circ} \mathrm{C}$ pendant 5 à $30 \mathrm{~min}$ ). Ces composés chimiques (des accélérateurs, des agents de traitement, des antioxydants, des stabilisateurs, des teintures, des décolorants et des agents explosifs) donnent au caoutchouc naturel ses propriétés finales telles sa couleur, sa texture et son élasticité. Le produit final est fait de 2 à $3 \%$ de protéines. Ces protéines solubles dans l'eau sont résistantes à la chaleur et à plusieurs agents chimiques. Le latex est utilisé dans la fabrication de plus de 40000 produits dont plusieurs instruments médicaux incluant des gants, des 
cathéters urinaires, des condoms, des tubes endotrachéaux, des masques anesthésiques, des montures dentaires, des ballonnets pour lavement baryté, des sacs de stomie et des bouchons d'arrêt sur ligne intra-veineuse.

Il est bien connu que le latex peut causer une dermatite de contact. Cette dermatite est due à une réaction allergique d'hypersensibilité retardée (type 4), impliquant les lymphocytes T. Les produits chimiques de petit poids moléculaire (accélérateurs et antioxydants) ajoutés au latex durant sa transformation agissent comme des haptènes et stimulent les lymphocytes $T$ sensibilisés afin de déclencher la prolifération d'autres lymphocytes et monocytes. Ceci entraîne une inflammation des tissus et crée la dermatite.'

C'est seulement en 1979 que le premier cas d'urticaire de contact dû au latex fut décrit. ${ }^{2}$ Depuis ce temps, on a démontré que le latex ne causait pas seulement de l'urticaire de contact, mais aussi de l'urticaire généralisé, de l'asthme, et même de l'anaphylaxie. Cette dernière réaction allergique sévère fut décrite pour la première fois en $1987,{ }^{3}$ et plus de 10 décès lui ont été attribués depuis ce temps. ${ }^{4}$ La cause de ces réactions est un anticorps IgE contre une ou plusieurs protéines dans le latex. ${ }^{5}$ Chez les patients qui possèdent cet anticorps, une exposition au latex peut provoquer une réaction d'hypersensibilité allergique immédiate (type 1), avec dégranulation des mastocytes et une anaphylaxie potentiellement dangereuse pour la vie.

Certains groupes de personnes sont plus à risque de développer une réaction anaphylactique au latex. Parmi ces personnes, il y a les travailleurs du domaine de la santé, particulièrement ceux qui portent fréquemment des gants de latex. Des études ont démontré que 6 à $10 \%$ du personnel oeuvrant en salle d'opération ont des anticorps IgE contre les protéines contenues dans le latex. ${ }^{6.7}$ Les enfants avec myéloméningocèle ou anomalies urologiques congénitales représente un autre groupe à risque, ${ }^{8-10}$ peut-être même le groupe le plus à risque de présenter des réactions sévères au latex. Au cours d'une étude faite dans cinq cliniques de myélodysplasie, un questionnaire distribué auprès de 187 familles a permis d'établir que $25 \%$ des enfants avec myéloméningocèle avait une histoire positive d'allergie aux produits de latex." D'autres études ont démontré que $56 \%$ des patients ( 71 sur 127 ) avec myéloméningocèle avaient des anticorps IgE contre le latex. ${ }^{12-14}$ Il est possible que l'exposition à répétition aux produits de latex (au cours des cathété-rismes urinaires et des chirurgies multiples) durant le jeune âge de ces patients entraîne une sensibilisation au latex.

Les patients avec antécédents d'atopie (rhinite allergique, asthme ou dermatite atopique) sont aussi plus à risque de développer une allergie au latex. ${ }^{8,9,15}$ De plus, les individus avec eczéma des mains et exposition aux gants de latex sont à haut risque de développer une réaction anaphylactique au latex. ${ }^{15,16} \mathrm{Ceci}$ s'explique peut-être par le fait qu'une peau eczémateuse perd son étanchéité et permet un contact plus facile entre les antigènes du latex et les cellules immunocompétentes sous l'épiderme. D'autre part, il faut aussi considérer que l'eczéma peut être une manifestation précoce d'allergie au latex chez ces patients. Toutes ces hypothèses restent à élucider. Notons que les patients allergiques aux bananes sont aussi des sujets à risque d'allergie au latex. $^{17}$

Que peut-on faire pour minimiser le risque des réactions allergiques au latex durant l'anesthésie? D'abord, il faut faire connaître aux cliniciens l'existence de cette allergie et leur montrer à identifier les groupes à risque. Des questions précises concernant des réactions antérieures au latex ou aux produits de caoutchouc doivent faire partie du questionnaire pré-anesthésique. ${ }^{18}$ Les patients qui présentent une réaction systémique sévère au latex pendant une chirurgie ont souvent des antécédents d'urticaire ou d'angio-oedème suite à une exposition à des produits de caoutchouc tels des gants, des ballons, des condoms, des diaphragmes ou des sucettes. L'allergie au latex doit aussi être suspectée chez les patients avec une histoire antérieure d'anaphylaxie peropératoire inexpliquée. Une étude récente a démontré que l'allergie au latex était responsable de 5 des 43 cas d'anaphylaxie peropératoire dans une même institution. ${ }^{19}$ L'anaphylaxie aux agents anesthésiques survient surtout au début de l'anesthésie, alors que l'anaphylaxie au latex survient le plus souvent 20 à 150 minutes après l'induction anesthésique, probablement au moment où les mains gantées touchent les muqueuses et le péritoine pour ainsi permettre l'absorption des protéines du latex. ${ }^{20}$

Le diagnostic d'allergie au latex se doit d'être confirmé par des tests épicutanés. On peut utiliser soit du latex naturel provenant de compagnies produisant du latex, ou une préparation faite à partir de gants de latex incubé dans une solution saline avec un tampon de phosphate, ou des tests cutanés à travers un gant de latex humide. ${ }^{16,21,22}$ Un réactif pour test cutané (latex naturel) est maintenant disponible au Canada (Bencard Lab., Mississauga, Ontario). A moins d'être familier avec tous les aspects de l'allergologie, il est préférable de référer les patients à un allergiste qui saura faire et interpréter ces tests cutanés. Les tests épicutanés superficiels avec large spectre dilutionnel sont très sécuritaires et permettent d'établir une sensibilité proportionnelle à la dose.

Les tests intradermiques sont rarement nécessaires et peuvent provoquer des réactions systémiques chez certains patients. Un test de provocation où l'individu porte un gant de latex humidifié a déjà causé des réactions systémiques et n'est donc pas recommandé. Le 
«RAST » («radioallergosorbent test ») pour le latex est aussi disponible (Latex disc 82, Pharmacia Diagnostics, Piscataway, NJ), mais il est moins sensible que les tests épicutanés. En fait, le RAST peut être négatif chez 20 à $40 \%$ des patients avec des tests cutanés positifs. ${ }^{15.23}$

Chez le patient chirurgical avec une sensibilité connue au latex, ou avec une histoire franche de réaction au latex, il faut absolument éviter l'utilisation de produit fait de latex, particulièrement les gants. Peut-être faudrait-il aussi éviter l'utilisation de ces produits chez les patients avec myélodysplasie ou anomalies urologiques congénitales, car la prévalence d'allergie au latex est élevée chez ces patients.

Le port des gants de latex peut être remplacé par le port de gants de caoutchouc synthétique fait de matériaux tels le «Neolon» (Deseret Medical, Inc.) ou «l'Elastyren» (Hermal Pharmaceutical Labs, Inc.). Cependant, ces gants de caoutchouc synthétique sont chers et ne sont pas toujours disponibles. De plus leurs propriétés physiques sont peu appréciées des utilisateurs. Pour ces raisons, le personnel médical responsable d'un patient allergique au latex peut préférer utiliser des gants de vinyle par-dessus des gants de latex lavés. Il a même été suggéré qu'un bon nettoyage des gants de latex avant leur utilisation chez le patient allergique suffit pour enlever les allergènes. ${ }^{22}$ Cependant, d'autres études sont nécessaires afin de confirmer la valeur de cette pratique avant d'en faire une recommandation.

Un traitement prophylactique avec des stéroïdes et des antihistaminiques a été suggéré pour prévenir les réactions d'hypersensibilité chez les patients allergiques au latex. Cependant l'efficacité d'une telle pratique n'a pas été prouvée. La valeur des stéroïdes et des antihistamines dans la prévention des allergies dues à des anticorps $\operatorname{IgE}$ reste à démontrer. Ces médicaments peuvent masquer les signes précoces d'une anaphylaxie et pour cette raison, leur utilisation n'est généralement pas recommandée. L'anesthésiste doit être à l'affût des premiers signes d'anaphylaxie et doit être prêt à les traiter agressivement.

Les patients allergiques au latex doivent informer de leur allergie toutes les personnes qui ont à les soigner. Ces patients devraient porter une identification «MedicAlert. » Ils devraient aussi avoir avec eux une seringue «Epi Pen» et apprendre à s'en servir. De plus, ils devraient avoir avec eux des gants faits de matériel synthétique afin d'assurer leur disponibilité pour le personnel traitant en cas d'urgence.

Bien que les produits faits de latex et de caoutchouc soient utilisés depuis longtemps, c'est seulement au cours de la dernière décennie que nous avons vu apparaître des cas d'urticaire de contact et de réaction anaphylactique systémique. Peut-être que les cliniciens n'ont pas perçu la relation entre la réaction allergique et l'exposition au latex dans le passé, mais ceci est peu probable. Il est aussi permis de croire que l'utilisation accrue des produits de latex, particulièrement les gants et les condoms, suite à la découverte du virus HIV, ait entraîné une plus grande exposition et une sensibilisation d'une plus grande population. Il est aussi possible qu'un changement dans la transformation manufacturière ait causé une plus grande antigénicité des produits de latex.

\section{References}

1 Adams RM. Diagnosis of allergic contact dermatitis of occupational origin. Clin Rev Allergy 1986; 4: 323-38

2 Nutter AF. Contact urticaria to rubber. $\mathrm{Br} J$ Dermatol 1979; 101: 597-8.

3 Axelsson JGK, Johansson SGO, Wrangsjo K. IgE-mediated anaphylactoid reactions to rubber. Allergy $1987 ; 42$ : 46-50.

4 Gelfand DW. Barium enemas, latex balloons, and anaphylactic reactions. Am J Radiol 1991; 156: 1-2.

5 Slater JE. Rubber anaphylaxis. N Engl J Med 1989; 320: 1126-30.

6 Turjanmaa $K$, Reunala $T$. Contact urticaria from rubber gloves. Derm Clin 1988; 6: 47-51.

7 Turjanmaa $K$. Incidence of immediate allergy to latex gloves in hospital personnel. Cont Derm 1987; 270-5.

8 Kelly $K$. Setlock M, Davis JP. Anaphylactic reactions during general anesthesia among pediatric patients - U.S., January 1990 - January 1991. MMWR 1991; 40: 437-43.

9 Sussman GL, Turlo S, Dolovicht J. The spectrum of IgEmediated responses to latex. JAMA 1991; 265: 2844-7.

10 Nguyen DH, Burns MW, Shapiro GC, Maho ME, Murrey $M$, Mitchell $M E$. Intraoperative cardiovascular collapse secondary to latex allergy. J Urol 1991; 146: 571-4.

11 Meeropol E, Kelleher $R$, Bell $S$, Leger $R$. Allergic reactions to rubber in patients with myelodysplasia. N Engl J Med 1990; 323: 1072.

12 Sandberg ET, Slater JE, Roth DR, Abramson SL. Rubberspecific IgE in children in a spina bifida clinic. J Allergy Clin Immunol 1992; 89: 223 (Abstract).

13 Yassin MS, Sanyurah S, Lierl MB, et al. Evaluation of latex allergy in patients with meningomyelocele. J Allergy Clin Immunol 1992; 89: 225 (Abstract).

14 Mathew SN, Melton A, Wagner W, Battisto JR. Latex hypersensitivity: prevalence among children with spina bifida and immunoblotting identification of latex proteins. J Allergy Clin Immunol 1992; 89: 225 (Abstract).

15 Wrangsjo $K$, Wahlbert $J E$, Axelssont $G K$. IgE-mediated allergy to natural nubber in 30 patients with contact urticaria. Cont Derm 1988; 19: 264-71.

16 Turjanmaa K, Laurila K, Mukinen-Kiljunen S, Reunala $T$. Rubber contact urticaria: allergenic properties of 19 brands of latex gloves. Cont Derm 1988; 19: 362-7. 
17 M'Raihi L, Carpin D, Bongrand P. Cross-reactivity between latex and banana. J Allergy Clin Immunol 1991; 129: 130.

18 Weiss ME, Adkinson NF Jr., Hirshman CA. Evaluation of allergic drug reactions in the perioperative period. Anesthesiology 1989; 71: 483-6.

19 Leynadier F, Pecquet C, Dry J. Anaphylaxis to latex during surgery. Anaesthesia $1989 ; 44: 547-50$.

20 Gold M, Swartz JS, Braude BM, Dolovich J, Shandling B, Gilmour $R F$. Interoperative anaphylaxis: an association with latex sensitivity. J Allergy Clin Immunol 1991; 87: 662-66.

21 Ownby DR, Tomlanovich M, Sammons N, McCullough J. Anaphylaxis associated with latex allergy during barium enema examinations. Am J Radiol 1991; 156: 903-8.

22 Morales C, Basomba J, Carreira, Sastre A. Anaphylaxis produced by rubber glove contact. Case reports and immunological identification of the antigens involved. Clin Exp Allergy 1990; 19: 425-30.

23 Turjanmaa K, Reunala T, Rasanen L. Comparison of diagnostic methods in latex surgical glove contact urticaria. Cont Derm 1988; 19: 241-7. 